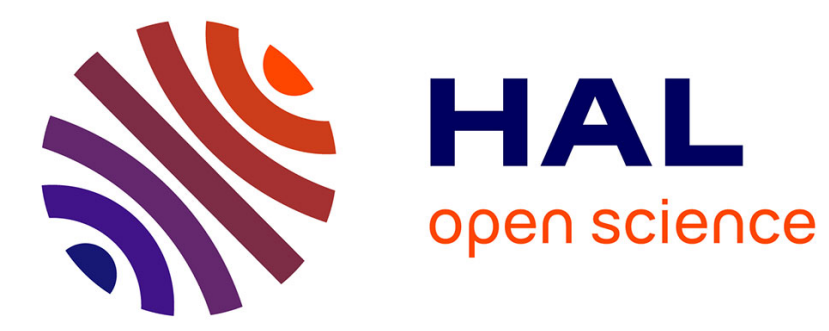

\title{
From regularity to chaos: a direct experimental test using atoms in magnetic field
}

N. Bouloufa, P. Cacciani, D. Delande, C. Delsart, J. Gay, E. Luc-Koenig, J. Pinard

\section{- To cite this version:}

N. Bouloufa, P. Cacciani, D. Delande, C. Delsart, J. Gay, et al.. From regularity to chaos: a direct experimental test using atoms in magnetic field. Journal de Physique II, 1992, 2 (4), pp.671-681. 10.1051/jp2:1992158 . jpa-00247664

\section{HAL Id: jpa-00247664 https://hal.science/jpa-00247664}

Submitted on 1 Jan 1992

HAL is a multi-disciplinary open access archive for the deposit and dissemination of scientific research documents, whether they are published or not. The documents may come from teaching and research institutions in France or abroad, or from public or private research centers.
L'archive ouverte pluridisciplinaire HAL, est destinée au dépôt et à la diffusion de documents scientifiques de niveau recherche, publiés ou non, émanant des établissements d'enseignement et de recherche français ou étrangers, des laboratoires publics ou privés. 
Classification

Physics Abstracts

$32.60-32.80-05.45$

\title{
From regularity to chaos: a direct experimental test using atoms in magnetic field
}

\author{
N. Bouloufa $\left({ }^{1}\right)$, P.Cacciani $\left({ }^{1}\right)$, D. Delande $\left({ }^{2}\right)$, C.Delsart $\left({ }^{1}\right)$, J.C. Gay $\left({ }^{2}\right)$, E. Luc-Koenig $\left({ }^{1}\right)$ \\ and J. Pinard $\left({ }^{1}\right)$
}

( $\left.{ }^{1}\right)$ Laboratoire Aimé Cotton, C.N.R.S., II, Bâtiment 505, 91405 Orsay Cedex, France

$\left({ }^{2}\right)$ Laboratoire de Spectroscopie Hertzienne de l'E.N.S., 4 place Jussieu, Tour 12.E1, 75252 Paris Cedex 05, France

(Received 6 December 1991, accepted 17 January 1992)

\begin{abstract}
Résumé . - Une expérience de spectroscopie a été réalisée sur des atomes de lithium plongés dans un fort champ magnétique $(4.9 \mathrm{~T})$ en utilisant une excitation laser à plusieurs étages des états proches de la limite d'ionisation. Un schéma d'excitation particulièrement bien choisi permet de tester sélectivement la symétrie dynamique des états excités. Cette expérience nous a permis d'observer, pour la première fois, la transition vers le chaos, comme résultant d'une brisure de ces symétries. La comparaison entre le spectre expérimental enregistré et le spectre calculé pour l'hydrogène montre un remarquablement bon accord.
\end{abstract}

\begin{abstract}
A spectroscopy experiment has been performed on lithium atoms in the presence of a strong magnetic field ( $4.9 \mathrm{~T})$ using multistep laser excitation of high lying levels. In this experiment, the peculiar excitation scheme is designed in order to selectively tests the dynamical symmetry of the excited states. This allows the direct observation of the transition to chaos from the breaking of these symmetries. The comparison between the experimental spectrum and a calculated one for hydrogen shows a remarkably good agreement.
\end{abstract}

\section{Introduction.}

During the past two decades, our understanding of the interaction of atoms with static field has greatly benefited from the studies led on Rydberg atoms. In such atoms, the valence electron is so weakly bound that external forces acting on it (electric, magnetic or even electromagnetic) become easily comparable or even greater than the Coulomb force. Theoretical studies of such systems cannot be based on a perturbative treatment but rather require a reformulation of the problem making full use of its peculiar symmetry properties, therefore looking for appropriate new constants of motion, separability. As a well known example, the theoretical analysis of 
Rydberg hydrogen atom in electric field was greatly helped and simplified by the existence of a system of coordinates in which the Hamiltonian is separable. In the mid seventies, numerous experimental studies on this subject were made possible thanks to the recent discovery of the wavelength tunable dye laser. They were performed mainly on alkali atoms and the analysis of spectra, in particular in the region where discrete and continuum states coexist, had revealed numerous unexpected new features. A review paper devoted to this fascinating subject was made by Jacquinot and Feneuille in 1981 [1].

In spite of ten years work, the more intricate problem of Rydberg atoms in interaction with a magnetic field still challenges our understanding. Due to the presence of the diamagnetic term: $\frac{\gamma^{2}}{8} \rho^{2}$ (in atomic units $\gamma=B / B_{0}, B_{0}=2.35 \times 10^{5} \mathrm{~T}, \rho$ the cylindrical radius), the Hamiltonian of the system becomes non-integrable and only approximate constants of motion can be found at low field strengths. Furthermore it has been demonstrated that, at high field, the classical motion of the electron becomes chaotic [2] as shown in figure 1 [3]. Rydberg atoms in a $\mathbf{B}$ field are certainly the simplest atomic system in which chaos can be observed and studied. Being a quantum system, this raises the question of how does the existence of chaos in the classical dynamics affects the quantum spectrum of the system. A criterion for characterizing "quantum chaos" has been found many years ago and has been applied in nuclear physics [4]: chaotic behaviour in the motion of the nucleus is characterized by peculiar statistical properties of the energy level spacings; this statistics (Wigner type) precludes the existence of small spacings (level repulsion) while the Poisson's one (which is the signature of a regular motion) is peaked at zero level spacing. This law can be easily adapted to atomic spectra. It has been checked successfully on theoretical spectra [5], but its application to the analysis of experimental data still remains hazardous: it actually requires the observation of all the levels even those associated with vanishingly small lines; such a study was performed, but only on the part of the spectrum in the regular regime, and a Poisson distribution was observed [6].

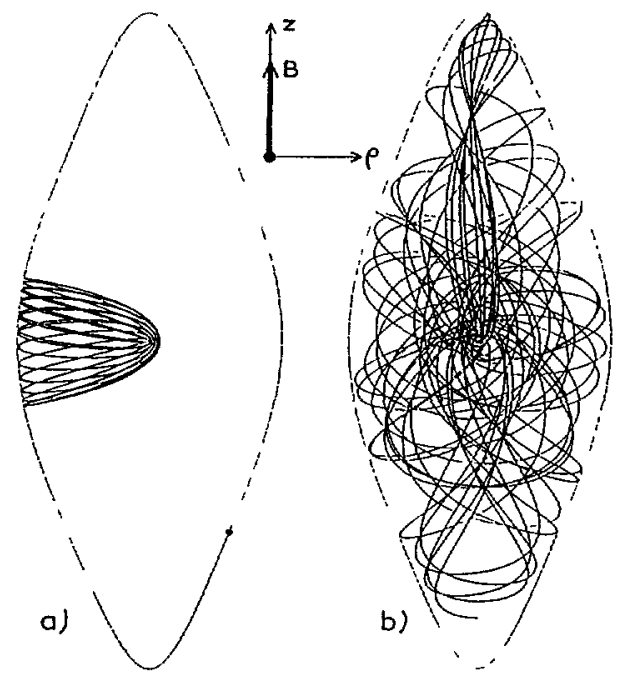

Fig. 1. - Two typical classical trajectories of an electron submitted to the combined Coulomb and magnetic forces (projection in the $\rho, z$ plane). a) regular motion, b) irregular (chaotic) motion. 
Indeed, the chaotic behaviour which follows from the lacking of a constant of motion (or a good quantum number in the quantal language) leads to an unexpectedly dense excitation spectrum. Nevertheless, the distinction between regular and chaotic spectra is not always obvious due to the fact that the symmetries of optical excitation do not necessarily comply with the dynamical ones of the system. To enhance this difference and unravel such intricate spectrum, it is possible to take advantage of the symmetry properties of the system: by selecting states with a well definite dynamical symmetry, the excitation spectrum will be simple and less dense in the regular region, and the modification due to the onset of chaos will be more striking. In a classical language, this corresponds to the exploration of a limited region of the phase space. The experiment reported hereafter was designed in this spirit.

\section{Symmetry considerations.}

Ten years ago, it was demonstrated that two different limiting symmetries coexist in the diamagnetic multiplet in the region of inter-l mixing regime (the $B$ field being low enough to avoid $n$-mixing); this is associated to the existence of an approximate constant of motion valid up to the 4 th order in $B$ :

$$
\Lambda=4 \mathbf{A}^{2}-5 A_{z}^{2}
$$

(A is the Runge-Lenz vector) [7].

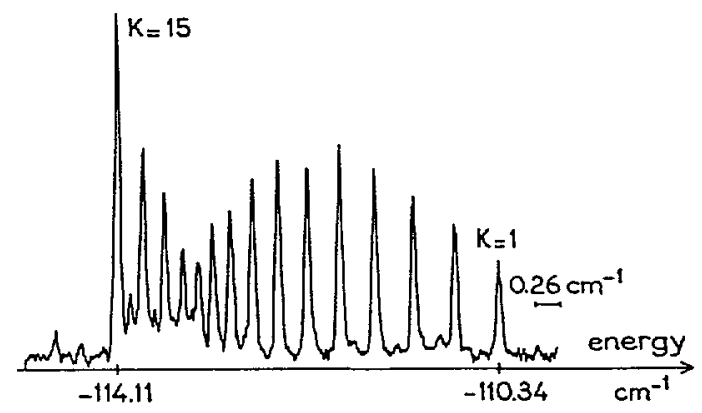

Fig. 2. - Experimental spectrum of the $n=30$, odd parity, diamagnetic multiplet of $\mathrm{Li}$ atom $(B=1.65 \mathrm{~T})$.

A recording of such diamagnetic multiplet is shown in figure 2. One can easily observe two different structures separated by a cross-over [8]:

In the low energy part of the spectrum, the corresponding states have negative $\Lambda$ values and the $\mathbf{A}$ vector lies near the $Z$ axis. For these states, $A_{z}$ is also an approximate constant of motion and they behave as Stark states; they are well described by parabolic wavefunctions. The symmetry is said to be of "vibrational" type [9]. Classically, the electronic motion is localized in the vicinity of the $Z$ axis. In phase space, the corresponding trajectories lie on tori surrounding the stable $\rho=0$ trajectory.

For the high energy part, the $\Lambda$ values are positive and the $\mathbf{A}$ vector is localised near the $Z=0$ plane. The states are said to have a "rotational" symmetry character. They are approximately eigenstates of $\lambda^{2}=A_{x}^{\prime 2}+A_{y}^{\prime 2}+L_{z}^{2}$ (where $A_{x}^{\prime}, A_{y}^{\prime}$ are the components of 

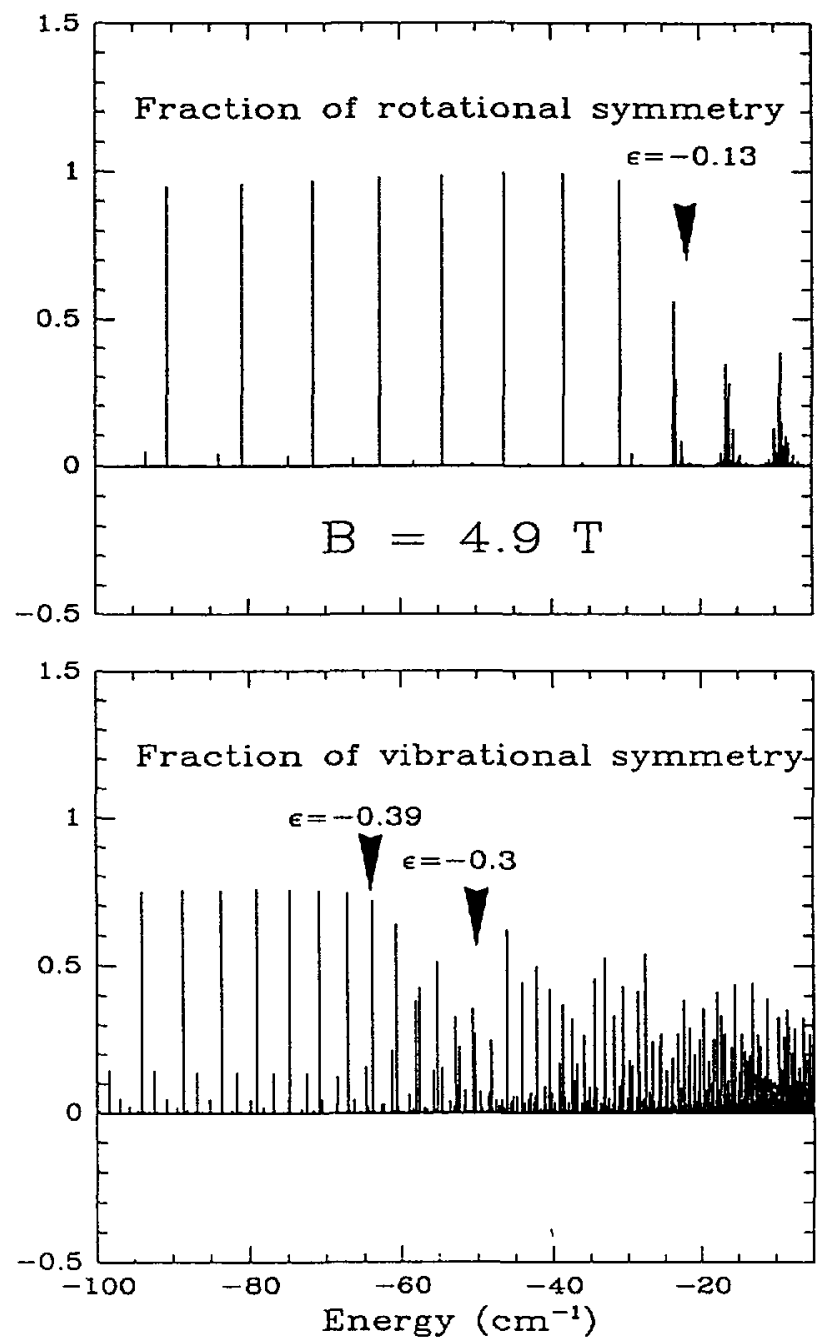

Fig. 3. - Simulations of the $M=0$, even parity spectrum of hydrogen atom. Classical onset of chaos appears for $\varepsilon=-0.13$ in the case of rotational symmetry spectrum and in the $\varepsilon$ range $[-0.39,-0.3]$ in the case of vibrational symmetry spectrum.

the scaled Runge Lenz vector and $L_{z}$ the $Z$ component of the orbital angular momentum). Classically, the electron moves in the vicinity of the $Z=0$ plane. In the phase space, the corresponding trajectories lie on tori surrounding the stable $Z=0$ trajectory. The higher energy states of the multiplet are well approximated by eigenfunctions of $\lambda^{2}$; they are said to have the $\lambda$-symmetry with $\lambda=n-1$ for the highest state of a $n$-multiplet. This symmetry properties can be extracted from a more general treatment of the Hamiltonian expressed in terms of coupled harmonic oscillators [10].

At higher magnetic field, the classical dynamics exhibits a transition from (previously discussed) regular regime to chaos. Due to classical scaling properties, the classical dynamics only 
depends on the scaled energy $\varepsilon=\frac{E}{\gamma^{2 / 3}}$ where $E$ is the binding energy. Below $\varepsilon=-0.54$, it is mainly regular (either "rotational" or "vibrational"). A study of Poincaré surfaces of section shows that the classical chaos appears at $\varepsilon=-0.54$ close to the separatrix (at the cross-over between vibrational and rotational symmetries) and extends first to the vibrational domain for increasing values of $\varepsilon$ up to around -0.39 ; above this value, the $\rho=0$ orbit turns unstable, and some chaos appears in its vicinity. At $\varepsilon=-0.32$, the last "vibrational" tori are destroyed. For still higher $\varepsilon$ values the rotational tori begin to be destroyed, and the system is entirely chaotic for $\varepsilon=-0.13$.

One can study the destruction of the vibrational and rotational symmetries in the quantum spectrum by projecting all the eigenstates on the extreme vibrational $\left(A_{z}=n-1\right)$ or rotational $(\lambda=n-1)$ states [3]. One will obtain in the regular region a very sparse and regular spectrum almost composed of only one line per $n$-multiplet corresponding to a pure vibrational (respectively rotational) spectrum. In the chaotic region these dynamical symmetries are destroyed (at least partially) and, the oscillator strength is spread out over all the energy levels. Consequently, a high density of excitation lines must burst out. An exemple of such spectrum is shown in figure 3; the transition from the regular to the "irregular" regime is well defined and has been studied in connection with features of the classical dynamics [3]. One can notice that, on the figure 3 , the symmetry breaking appears around $\varepsilon=-0.13$, corresponding to the destruction of the most stable rotational torus.

The realisation of an experiment intending to record and study dynamical symmetry spectra is not easy using optical excitation: no selection rule exists in electric dipole excitation for the symmetry operators of interest $\left(A_{z}\right.$ or $\left.\lambda^{2}\right)$ and experimental plots as perfect as those in figure 3 cannot be obtained. Nevertheless one can show that the optical excitation from a state with well definite dynamical symmetry populates preferentially final states of the same symmetry. This holds true only if the lower states of the transitions are already diamagnetic in character and well isolated from each other. That is the principle of the experiment we describe now.

\section{The experimental scheme.}

The experiment was performed on an atomic beam of lithium and, as mentioned above, in order to excite a symmetry selected spectrum, the lower state of the transitions is chosen as corresponding to a definite state of an already resolved diamagnetic manifold. It is convenient to use, as such an intermediate state, one component of the $n=10$ multiplet: the $n$ value is large enough so as to achieve a sufficient level spacing and not too high as to permit further optical excitation with single mode, tunable laser sources, in a frequency range still relatively easy to reach $(10 \mu \mathrm{m})$. For the excitation from the lower level of the $n=10$ multiplet, the quantum defect of $\mathrm{Li}$, though small, has a significant effect: an efficient level population can only be obtained if the $10 \mathrm{~d}$ excited state is strongly mixed with the other components of the multiplet. We have chosen to perform a two-step excitation of the $d$ state via the resonant $2 p$ level, combined with a Stark switching process which increases noticeably the mixing and permits an excitation of all the components of the multiplet. Figure 4 represents the three-step excitation scheme. The recording of the intermediate $n=10$ manifold for $B=4.9 \mathrm{~T}$ in the presence of an electric field of $200 \mathrm{~V} / \mathrm{cm}$ (Fig. 5) underlines the efficiency of the mixing.

The experimental set-up is presented in figure 6; the atomic beam of lithium crosses the interaction region located at the center of a superconducting magnet (Helmoltz split-coil type); 


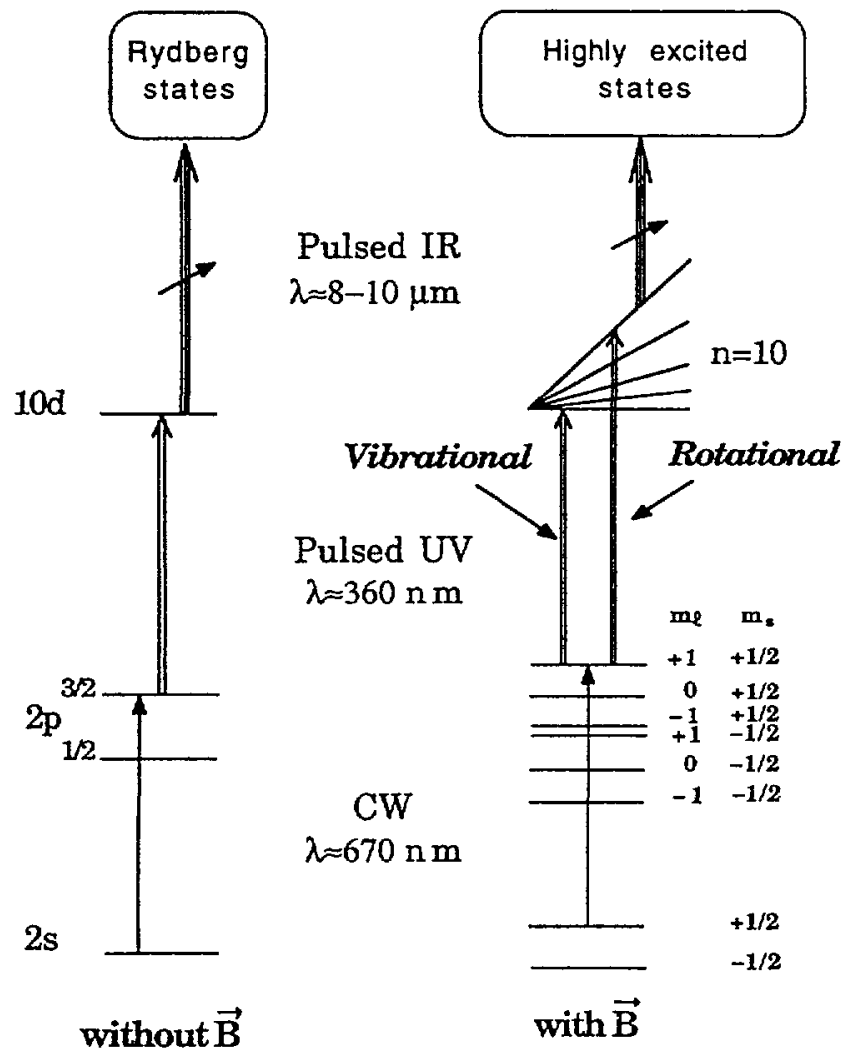

Fig. 4. - Excitation scheme for the recording of a symmetry selected spectrum.

in this region an electric field can be applied in the direction of the $B$-field in order to either perform the Stark switching process or to detect the populated high-lying states using the electric field ionisation method. Then the yield of electrons is sent on a surface barrier detector which gives the recorded signal after passing through a digital oscilloscope for fast digitalization and averaging, all the data (signal and references) being stored using a PDP 11 computer.

The three-step excitation of lithium atoms is performed in the interaction region using three laser beams:

i) the first one is produced by a cw single-mode dye laser, it allows to populate one definite state of the Zeeman pattern of the resonance line at $670 \mathrm{~nm}$;

ii) the second one is pulsed, it is produced by a single mode laser system [11], its frequency $(720 \mathrm{~nm}$ ) is doubled in order to reach a component of the $n=10$ manifold (in the presence of an electric field as mentioned above);

iii) the basic device of the third beam, of the same type as the second one, consists of a powerful single mode, continuously tunable, pulsed dye laser system; it provides up to $50 \mathrm{~mJ}$ pulses at a wavelength around $750 \mathrm{~nm}$; this beam is then focused in a Raman cell filled with 


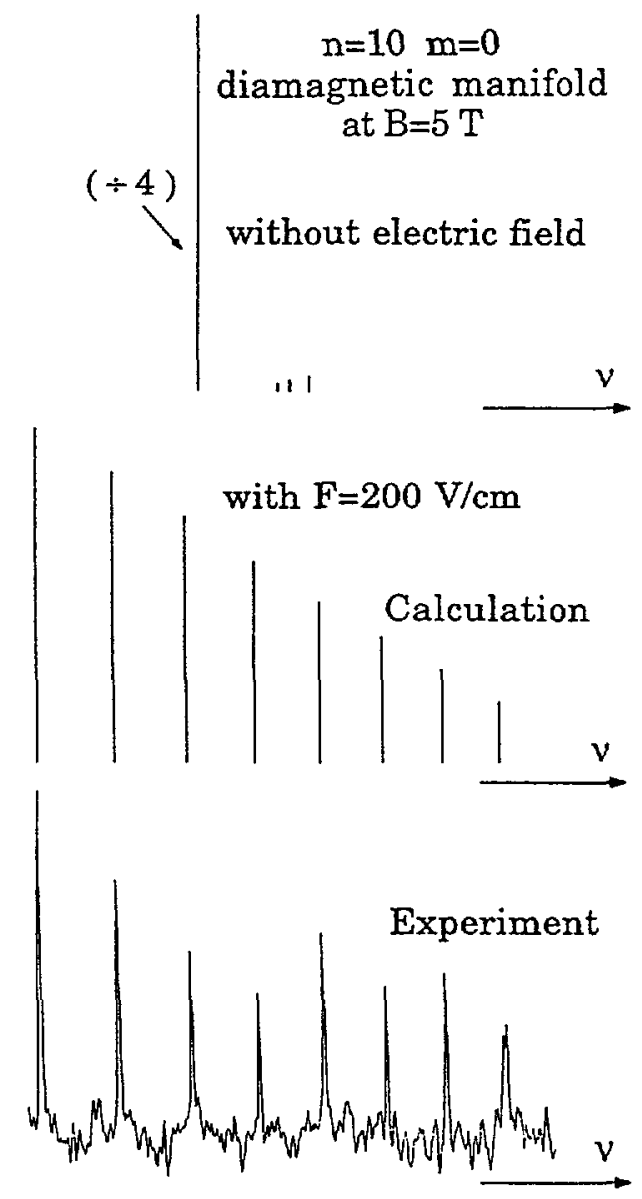

Fig. 5. - Calculated and experimental spectra of the intermediate $n=10$ manifold: influence of a parallel electric field added to the magnetic field.

15 bars of $\mathrm{H}_{2}$ in order to create through stimulated third Stokes Raman scattering a beam of light in the $9-10 \mu \mathrm{m}$ range; we use a capillary to canalize the input beam and thus increase the efficiency. Up to $10 \mu \mathrm{J}$ pulses have been obtained this way.

The first laser frequency is locked on the fluorescence signal of the atomic beam detected by a photomultiplier (P.M.). The two pulsed lasers are frequency stabilized and scanned using sigmameters [12] which insure, for the second step, a good long term stability. The timing of the experiment is such that the second step is applied in the presence of an electric field which is then decreased adiabatically to zero before the start of the final step, $150 \mathrm{~ns}$ later. Then a delayed $1 \mathrm{kV}$ pulse is applied, which allows the detection of the high lying excited states through field ionization.

In order to get an absolute frequency scale for the energy spectrum, a simultaneous recording of the iodine absorption spectrum is performed using a part of the fondamental beam of the frequency scanned laser. The excitation frequency is then deduced from the iodine reference frequency by just substracting three times the Raman shift: $4155 \mathrm{~cm}^{-1}$ 


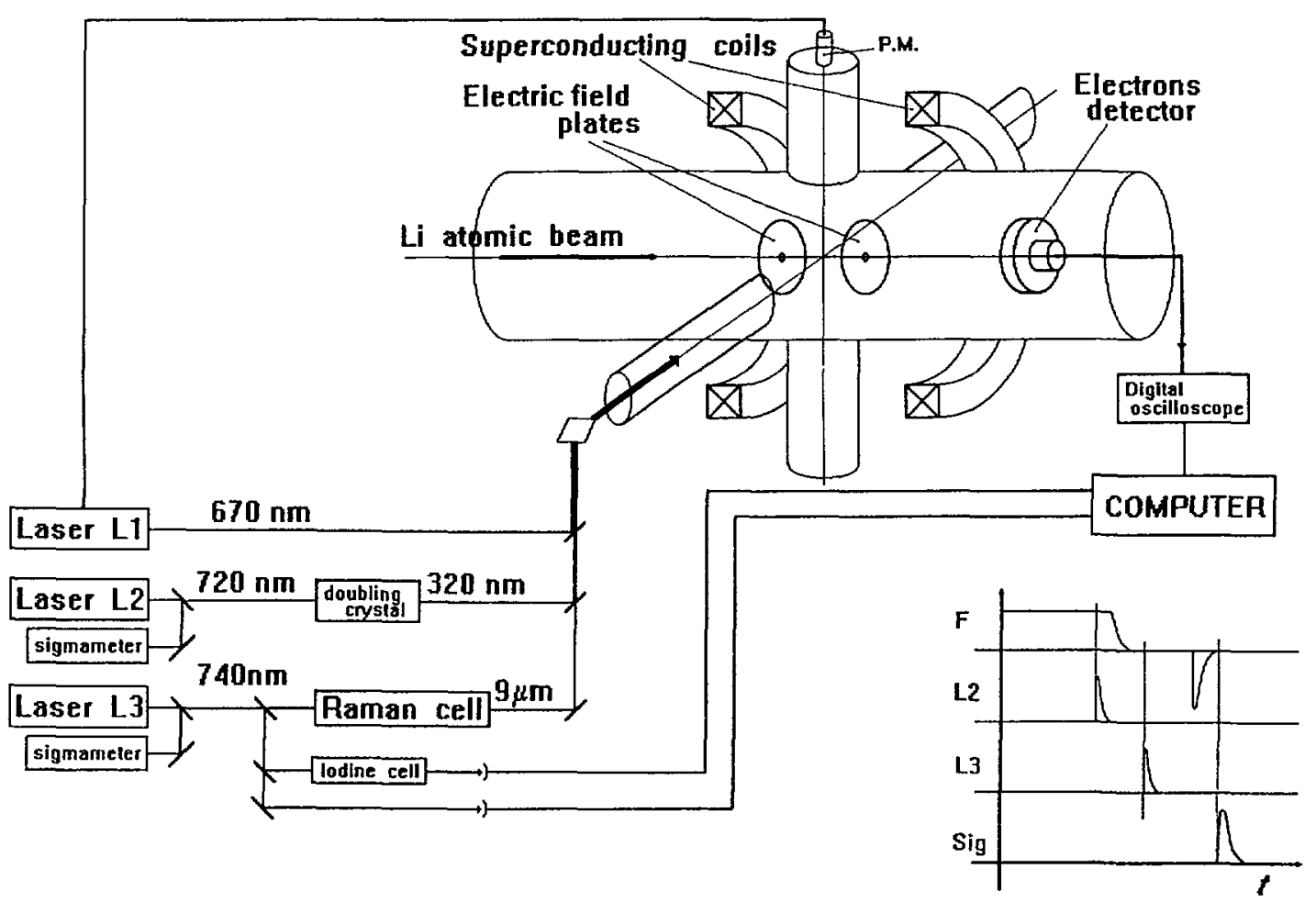

Fig. 6. - The experimental set up. The timing of the two laser pulses (L2 and L3) with respect to the applied electric field $F$ is shown in the lower right part of the figure.

\section{Experimental results.}

The spectrum will be strongly dependent on the parity and $m$-value of the lower state of the last transition as well as on the polarisations of the excitations, thus the three laser polarisations have to be conveniently selected; the choice is done by looking at the calculated spectrum of a non-mixed $n$-manifold. As an example, figure 7 presents the calculated spectrum obtained for an excitation of the lower vibrational state of the $n=10$ multiplet to the $n=22$ group, by using a laser polarisation scheme of the type: $\sigma, \pi, \pi$ for respectively, the first, the second, and the third step. It shows, in this particular case, how good is the symmetry selectivity in the excitation process: around $90 \%$ of the oscillator strength of the excited lines is included in the final states with vibrational symmetry. Furthermore, this choice allows us to record the selected $m=+1$ spectrum; this is not the case when, for example, the last excitation is of the $\sigma$ type: the laser beam configuration (transverse excitation of the atomic beam) does not permit to distinguish between $\Delta m=+1$ or -1 excitation, and two spectra split by the paramagnetic term appear superimposed, increasing the number of lines and the difficulties of interpretation.

Using the polarisation scheme mentioned above, a vibrational spectrum has been recorded in the frequency region where the transition to chaos in the classical system is expected to occur; the result is shown in figure 8 . In the same figure we have reproduced, at the same scale, a calculated spectrum obtained using a powerful diagonalisation method (Delande D. 


$$
(n=10, m=1, k=1) \stackrel{B=5 T}{-} \rightarrow(n=22, m=1)
$$

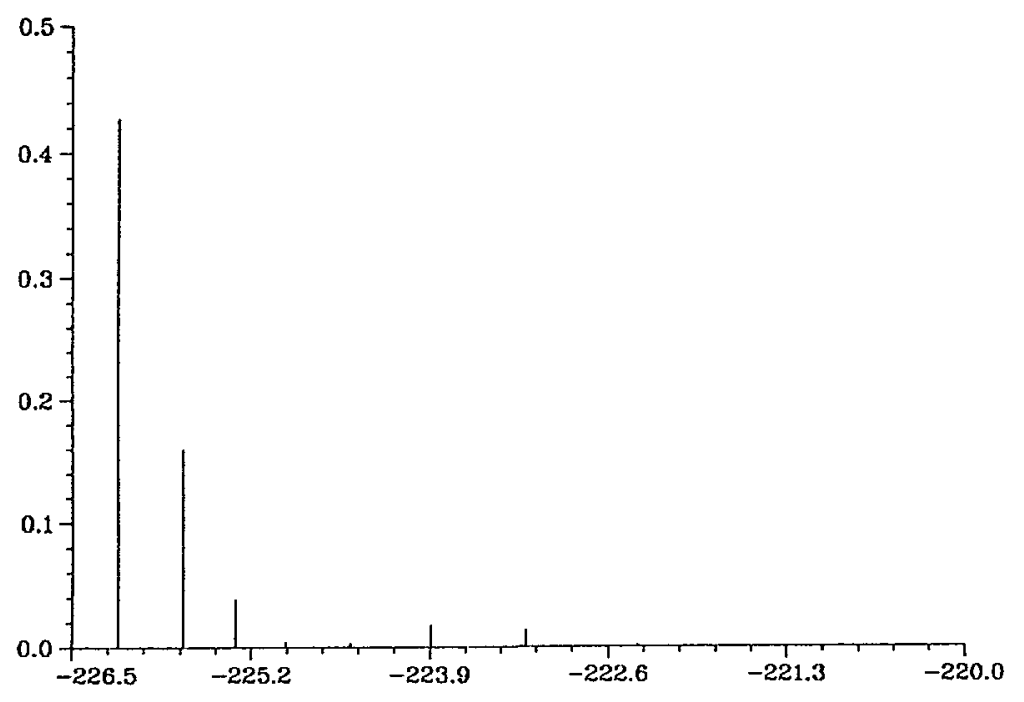

Fig. 7. - Calculated excitation spectrum of the $n=22$ manifold from the most vibrational state of the $n=10$ manifold and the $\sigma, \pi, \pi$ polarization scheme.

and Gay J.C. in Ref. [5]); this method allows a determination of the wavefunctions as well as the energies of the high lying levels in the pure hydrogen case. The core effect of lithium has been taken into account for the determination of the wavefunction of the lower state of the transition. For initial calculations, the value of the magnetic field strength used was experimentally determined from a measurement of the paramagnetic splitting of the 2p state; it was then refined by looking at the agreement between the experimental and calculated spectrum for a particular well recognizable structure: for example the one composed of two strong lines near $E=-72 \mathrm{~cm}^{-1}$, (the structure of the spectrum is extremely sensitive to the magnetic field in this chaotic region); we have determined: $B=4.915 \mathrm{~T}(\gamma=2.091)$.

The final result, plotted in figure 8 , shows a remarkably good agreement between the positions of lines for the whole experimental spectrum and the calculated ones; concerning the intensities, there is a discrepancy in the intensity ratio between strong and small lines, this can be attributed to saturation phenomena; the general broadening of the strong lines corroborates this statement. Nevertheless, the change in the spectrum corresponding to the onset of chaos is well observable, it is located around $E=-50 \mathrm{~cm}^{-1}$ (i.e. $\varepsilon=-0.3$ ); this value is slightly higher than the value generally associated to the destruction of all the classical tori with vibrational type symmetry $(\varepsilon=-0.39)$.

\section{Conclusion.}

For the first time, the energy spectrum of an atom perturbed by a strong magnetic field has been recorded in an energy range just below the zero field ionization threshold, in such a way that it allows a direct observation of a chaotic behaviour. The breaking of the symmetry which characterizes the onset of chaos is clearly seen, and unlike the other methods of characteri- 


\section{Lithium $\quad \mathrm{B}=4.9 \mathrm{~T} \quad$ Starting state: vibrational component}

$$
\mathrm{n}=10 \text { odd } \mathrm{M}=+1
$$
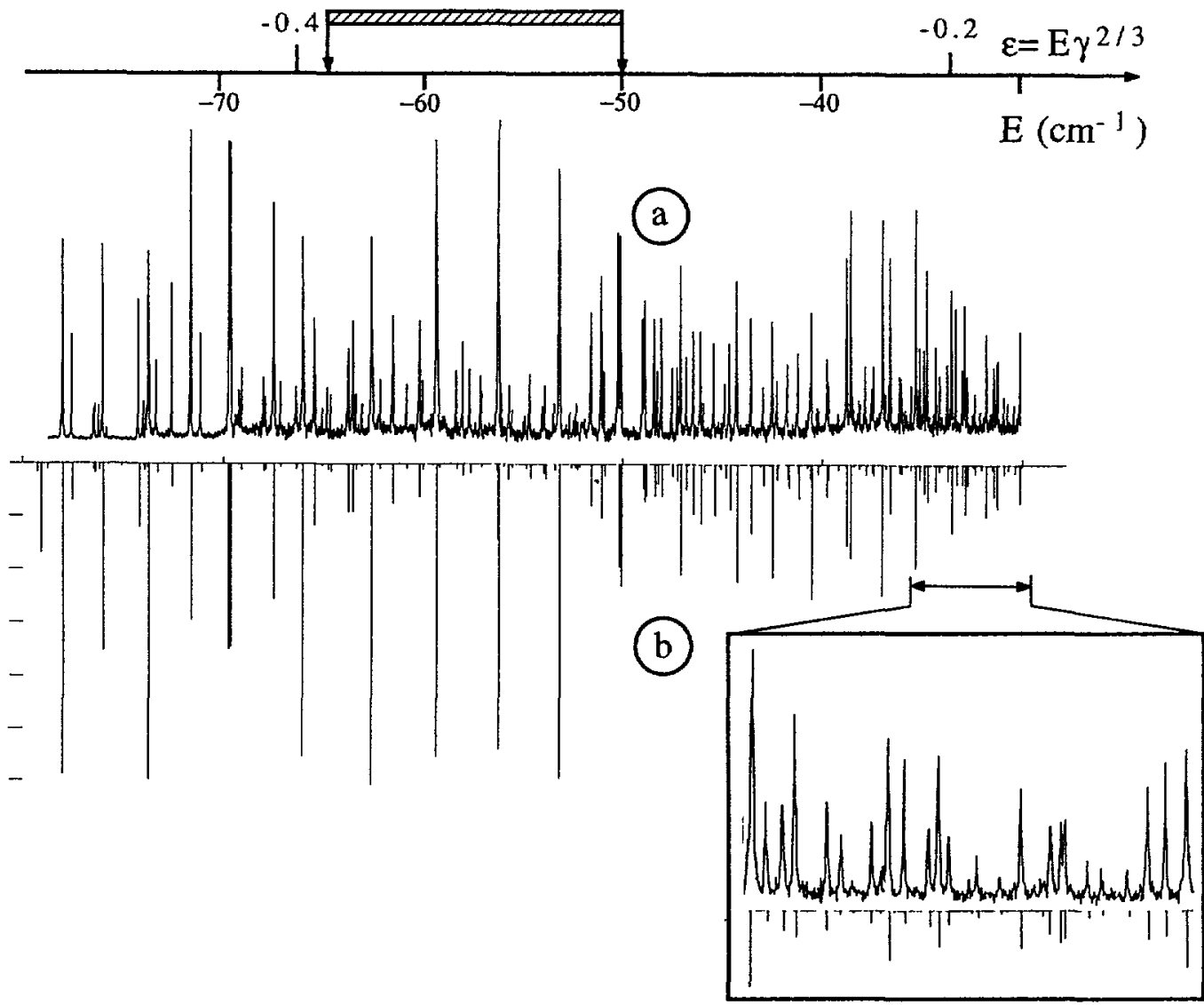

Fig. 8. - Recording of the vibrational, $m=1$, even parity spectrum in the frequency region surrounding the transition to chaos. a) experiment in lithium. b) comparison with numerical simulation on hydrogen.

zation which require a mathematical treatment of the experimental spectrum (e.g. through F.F.T [13]), the direct observation of our spectrum allows a determination of the energy of the transition at which the vibrational tori are destroyed.

This experiment was done by selecting states of vibrational symmetry; similar results are expected for the rotational symmetry selected spectrum, however the corresponding states are much more stable against chaos and the "breakdown" must appear at higher energy; this experimental situation is somewhat different from the one described above, it requires a different polarization scheme $(\pi, \pi \sigma)$ which makes a little more complicated, but not impossible, the interpretation of the spectrum; this experiment is in progress. 


\section{References}

[1] Feneullle S. and Jacquinot P., Adv. At. Mol. Phys. 17 (1981) 99.

[2] RoBnIK M., J. Phys. A 14 (1981) 3195;

Delos J.B., Knudson S.K. and NoID D.W., Phys. Rev. A 30 (1984) 1208.

[3] Delande D., These d'Etat, Paris (1988); in Chaos and Quantum Physics NATO, Les Houches, Lecture notes, 1989, A. Voros, M. Giannoni Eds (North Holland, 1990).

[4] Bohigas O., Giannoni M.J. and Schmit C., Phys. Rev. Lett. 52 (1984) 1.

[5] Wintgen D. and Friedrich H., Phys. Rev. Lett. 57 (1986) 571;

Delande D. and Gay J.C., Phys. Rev. Lett. 57 (1986) 2006;

WunNer G., WoelK U., Zech I., Zeller G., ERTL T., Geyer P., Schweitzer W. and RUDER P., Phys. Rev, Lett. 57 (1986) 3261.

[6] Welch G.R., KASh M.M., Chun-HO-IU, LoNg HsU and Kleppner D., Phys. Rev. Lett. 62 (1989) 893.

[7] SOLOV'EV E.A., JETP 34 (1981) 265.

[8] Cacciani P., Luc-Koenig E., Pinard J., Thomas C. and Liberman S., Phys. Rev. Lett. 56 (1986) 1124; J. Phys. B 21 (1988) 3473.

[9] Labarthe J.J., J. Phys. B 14 (1981) L-467;

Herrick D.R., Phys. Rev. A 26 (1982) 323.

[10] Delande D. and Gay J.C., Phys. Rev. Lett. 59 (1987) 1809.

[11] Pinard J. and Liberman S., Opt. Commun. 20 (1977) 344.

[12] Juncar P. and Pinard J., Opt. Commun. 14 (1975) 438.

[13] Holle A., Main J., Wiebusch G., Rottke H. and Welge K.H., Phys. Rev. Lett. 61 (1988) 161. 